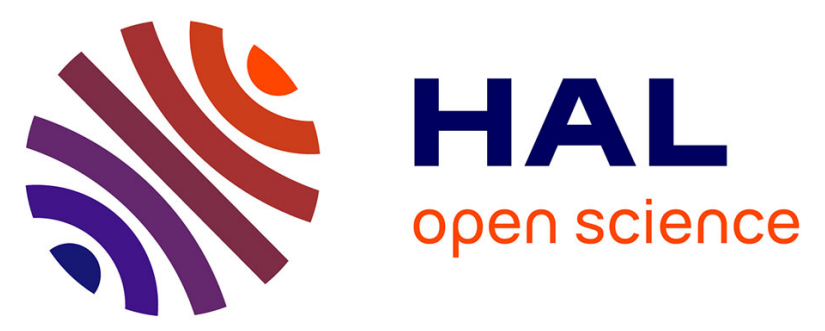

\title{
Derivation of a generalized double-sine-Gordon equation describing ultrashort-soliton propagation in optical media composed of multilevel atoms
}

\author{
Hervé Leblond, Houria Triki, Dumitru Mihalache
}

\section{- To cite this version:}

Hervé Leblond, Houria Triki, Dumitru Mihalache. Derivation of a generalized double-sine-Gordon equation describing ultrashort-soliton propagation in optical media composed of multilevel atoms. Physical Review A: Atomic, molecular, and optical physics [1990-2015], 2012, 86 (6), pp.063825. 10.1103/PhysRevA.86.063825 . hal-03187683

\section{HAL Id: hal-03187683 \\ https://univ-angers.hal.science/hal-03187683}

Submitted on 1 Apr 2021

HAL is a multi-disciplinary open access archive for the deposit and dissemination of scientific research documents, whether they are published or not. The documents may come from teaching and research institutions in France or abroad, or from public or private research centers.
L'archive ouverte pluridisciplinaire HAL, est destinée au dépôt et à la diffusion de documents scientifiques de niveau recherche, publiés ou non, émanant des établissements d'enseignement et de recherche français ou étrangers, des laboratoires publics ou privés. 


\title{
Derivation of a generalized double-sine-Gordon equation describing ultrashort-soliton propagation in optical media composed of multilevel atoms
}

\author{
Hervé Leblond, ${ }^{1}$ Houria Triki, ${ }^{2}$ and Dumitru Mihalache ${ }^{1,3,4}$ \\ ${ }^{1}$ LUNAM Université, Université d'Angers, Laboratoire de Photonique d'Angers, EA 4464, 2 Boulevard Lavoisier, \\ 49045 Angers Cedex 01, France \\ ${ }^{2}$ Radiation Physics Laboratory, Department of Physics, Faculty of Sciences, Badji Mokhtar University, P. O. Box 12, 23000 Annaba, Algeria \\ ${ }^{3}$ Academy of Romanian Scientists, 54 Splaiul Independentei, 050094 Bucharest, Romania \\ ${ }^{4}$ Horia Hulubei National Institute for Physics and Nuclear Engineering, 30 Reactorului, Magurele-Bucharest 077125, Romania
}

(Received 7 October 2012; published 20 December 2012)

\begin{abstract}
We consider the propagation of ultrashort optical solitons in media described by a general Hamiltonian of multilevel atoms. Assuming that all transition frequencies of the medium are well below the typical wave frequency, i.e., only the contribution of infrared transitions is taken into account, we use a short-wave approximation and a rigorous application of the reductive perturbation formalism to derive a cumbersome coupled system of nonlinear partial differential equations describing ultrashort soliton evolution in such systems. The rather complicated set of coupled equations can be simplified to a generic double-sine-Gordon equation for a special case of identical three-level atoms, whereas for a special case of identical four-level atoms the system of coupled equations can be reduced to a generalized double-sine-Gordon equation. Numerical simulations showing the formation of robust breather-type solutions of both the standard double-sine-Gordon and of the generalized double-sine-Gordon equations from sinusoidal inputs with Gaussian envelopes are also presented.
\end{abstract}

DOI: 10.1103/PhysRevA.86.063825

PACS number(s): 42.65.Tg, 42.65.Sf, 47.20.Ky

\section{INTRODUCTION}

Since the publication in 1999 by a few different research teams [1] of the pioneering works in the area of producing ultrashort optical pulses (in the two-cycle or even sub-twocycle regimes) by using Kerr-lens mode-locked Ti:sapphire lasers, this fertile research area evolved in many directions, ranging from light-matter interaction at ultrahigh peak powers and laser focused intensities [2], single-cycle nonlinear optics [3], and high-harmonic generation by ultrashort laser pulses and attosecond physics [4]; see also the reviews $[5,6]$ and two relevant works on the attosecond control of electronic processes by intense light fields [7] and on generation of giant half-cycle attosecond pulses [8]. We mention also other interesting recent developments in this very active research field: the synthesis of single-cycle light pulses by using compact erbium-doped fiber technology [9], the generation of ultrashort optical vortex pulses in the few-cycle regime [10], and the recent proposal (at a theoretical level) of a new amplification method, opening the way to exawatt-zetawatt level pulse generation [11].

The continuing experimental activity in the area of wave dynamics of ultrashort, few-cycle pulses (FCPs) in both the linear and nonlinear regimes has paved the way for the development of new theoretical models which adequately describe the propagation of ultrashort wave packets in a lot of relevant physical settings. Within the past two decades three classes of main dynamical models for FCPs have been put forward: (i) the quantum approaches [12], (ii) the refinements within the framework of slowly varying envelope approximations (SVEA) of the nonlinear Schrödinger-type envelope equations [13], and the non-SVEA models [14-28]. The propagation of FCPs in Kerr media can be described beyond the SVEA by using the modified Korteweg-de Vries $(\mathrm{mKdV})[20,21]$, sine-Gordon $(\mathrm{sG})$ [22,23], or mKdV-sG equations [24-26]. Note that a special case of $\mathrm{mKdV}-\mathrm{sG}$ equation is the so-called short-pulse equation (SPE) which was first introduced in Ref. [29], to describe FCP propagation in silica fibers. The SPE was later used in the study of localized structures in frequency band gaps of nonlinear metamaterials [30]. Recently, two different versions of the SPE in $(2+1)$ dimensions were studied in detail [31] and it was shown that ultrashort one-dimensional breathers appear to be fairly robust in the two-dimensional setting, while rather general two-dimensional localized initial conditions are transformed into quasi-one-dimensional dispersing wave forms, which are reminiscent of the one-dimensional solitons.

Though the SVEA is no longer valid for ultrashort optical pulses with duration of only a few femtoseconds, several generalizations of the SVEA have been proposed and have proven their efficiency. Note that these generalizations were referred to in the literature as higher-order nonlinear Schrödinger (NLS) models; see, e.g., Refs. [13]. Here we mention that first-order nonlinear evolution equations can be obtained under the socalled unidirectional approximation [32]. Non-SVEA models were proposed within the framework of the unidirectional approximation; see, e.g., Ref. [33]. However, to the best of our knowledge, the necessity of using the non-SVEA approach for the adequate description of FCPs was put forward in the early seminal work by Akhmediev, Mel'nikov, and Nazarkin published in 1989 [34]. In a subsequent paper published in 1990, Belenov and Nazarkin [14] obtained some exact solutions outside the approximation of slowly varying amplitudes and phases for light pulses a few wavelengths long and with high intensities, clearly stating that traditional SVEA methods "are becoming ineffective in describing wave processes at such small spatial and temporal scales and at such high fields." Moreover, in a recent work by Farnum and Kutz [35] on ultrafast pulse propagation in a mode-locked laser cavity in the few femtosecond pulse it was clearly stated that the standard NLS-based approach of ultrafast pulse propagation, though, 
has been shown "to work quantitatively beyond its expected breakdown, into the tens of femtoseconds regime, and has been used extensively for modeling supercontinuum generation, when pushed to the extreme of a few femtosecond pulses, the NLS description becomes suspect."

In the following we list a series of relevant recent works on the formation and dynamics of few-cycle pulses in a variety of physical settings: the propagation and interaction of extremely short electromagnetic pulses in quadratic nonlinear media [36], the study of few-cycle light bullets created by femtosecond filaments [37], the investigation of ultrashort spatiotemporal optical solitons in quadratic nonlinear media [38], the ultrashort spatiotemporal optical pulse propagation in cubic media without the use of the slowly varying envelope approximation [39], the possibility of generating few-cycle dissipative optical solitons [40], the generation of unipolar pulses from nonunipolar optical pulses in quadratic nonlinear media [41], the existence of guided optical solitons of femtosecond duration and nanoscopic mode area [42], and single-cycle gap solitons generated in resonant two-level dense media with a subwavelength structure [43]. Other recent theoretical works study a class of few-cycle elliptically polarized solitary waves in isotropic Kerr media [44] and robust circularly polarized few-optical-cycle solitons in Kerr media in both long-wave and short-wave approximation regimes [45]. Drozdov et al. [46] performed a comprehensive study of self-phase modulation and frequency generation with few-cycle optical pulses in nonlinear dispersive media. The nonlinear effects associated with the spatiotemporal propagation of few-cycle optical pulses in nonlinear dispersive media, including nonlinearity-induced self-phase modulation, generation of higher harmonics, and the effects of diffraction, were analyzed in detail [46]. A detailed study of ultrashort pulses and short-pulse equations in $(2+1)$ dimensions was also performed in a recent work by Shen et al. [31]. Two versions of the short pulse equation in $(2+1)$ dimensions were derived and by using Maxwell's equations as a starting point, and suitable Kramers-Kronig formulas for the permittivity and permeability of the medium, which are relevant, e.g., to left-handed metamaterials and dielectric slab waveguides, a multiple scales technique to obtain the relevant models was employed [31]. Kolesik et al. [47] quantified the limits of unidirectional ultrashort optical pulse propagation, explored the limits of the unidirectional pulse propagation equation in general nonlinear media, and investigated under which physical conditions two-way propagation becomes significant, and leads to a breakdown of the unidirectional approximation. Whalen et al. [48] studied optical shock and blowup of ultrashort pulses in transparent media and examined various ultrashort pulse propagation models and their relative effectiveness in explaining these phenomena. In particular, the modified Kadomtsev-Petviashvilli equation of type 1 was examined in some detail; see [48]. Another interesting recent theoretical study by Yan [49] deals with complex PT-symmetric extensions of the nonlinear ultrashort light pulse model. A family of interesting complex PT-symmetric extensions of the short pulse equation was presented and unique properties of these equations with some chosen parameters were studied; see Ref. [49]. In particular, Yan [49] obtained exact solitary wave solutions, doubly periodic wave solutions, and compacton solutions.
Most of the theoretical investigations concerned only FCPs propagating in nonlinear optical media described by two-level Hamiltonians. However, in two recent works [50,51] we extended the existing studies to a more general physical situation involving $N$-level Hamiltonians in the framework of the reductive perturbation method (multiscale analysis) [52]. First, in the long-wave approximation regime we gave in Ref. [50] a detailed mathematical derivation of the $\mathrm{mKdV}$ equation for a general $N$-level Hamiltonian. We assumed that the absorption spectrum of the nonlinear medium does not extend below some cutoff frequency, and that the typical frequency of the FCP is much less than the latter; therefore, a Kerr medium which has no transition in the infrared was actually described in Ref. [50]. Second, in the same long-wave approximation regime, we derived in Ref. [51] a coupled system of $\mathrm{KdV}$ equations describing ultrashort soliton propagation in quadratic media by using a general Hamiltonian for multilevel atoms. In Ref. [51] a detailed study of linear eigenpolarizations in the degenerate case and the corresponding formation of half cycle solitons from few-cycle-pulse inputs were discussed.

In the present work by using the reductive perturbation formalism (multiscale analysis) [52] we give a detailed mathematical derivation of the set of coupled nonlinear equations describing ultrashort soliton propagation by considering a general Hamiltonian for multilevel atoms in the short-wave approximation regime. Thus by taking into account the effect of the infrared transitions we are left with a rather cumbersome set of coupled partial differential equations. In two special physical situations this rather complicated set of coupled equations can be simplified to either a generic double-sineGordon equation (for a special case of a three-level system) or to a generalized double-sine-Gordon equation (for a special case of a four-level system). It is to be mentioned that in the simplest case of two-level identical atoms, this cumbersome set of equations reduces to a $\mathrm{sG}$ model, formally identical to that describing self-induced transparency (SIT), but in different validity conditions.

This paper is organized as follows. In the next section we present in detail the governing equations for the density matrix in the case of the most general Hamiltonian for multilevel atoms and we analyze the corresponding electromagnetic wave equations. We work in the so-called short-wave regime and we perform the multiscale analysis [52] order by order. In Sec. III we analyze in detail a first special case, assuming that a general four-level system may interact with the light wave only through two independent transitions. In this simplified situation we get a generalized double-sine-Gordon model. This approach straightforwardly generalizes to an arbitrary number of independent transitions. Then, in Sec. IV we consider a second special case, accounting for the coupling between two transitions with equal weights. Specifically, it deals with a general three-level system, in which the corresponding two excited levels are not coupled together. We are left in this special case with the generic doublesine-Gordon equation. In Sec. $\mathrm{V}$ we perform a detailed numerical study of breather-type solutions of both the standard double-sine-Gordon equation and the generalized doublesine-Gordon equation. We show that robust breather-type solutions can be formed from adequate inputs. Such breather solutions are in fact the sought-after prototype wave forms 
of ultrashort two-cycle solitons which form in the corresponding physical setting. Finally, in Sec. VI we present our conclusions.

\section{DERIVATION OF A GENERAL MODEL}

\section{A. Electromagnetic wave equation for a set of identical atoms}

The evolution of the electric field $\mathbf{E}$ is governed by the equation

$$
\Delta \mathbf{E}-\nabla(\boldsymbol{\nabla} \cdot \mathbf{E})=\frac{1}{c^{2}} \frac{\partial^{2}}{\partial t^{2}}\left(\mathbf{E}+\frac{1}{\varepsilon_{0}} \mathbf{P}\right),
$$

where $c$ is the light velocity in vacuum, $\Delta$ is the Laplacian operator, $\varepsilon_{0}$ is the dielectric permittivity of vacuum, and $\mathbf{P}$ is the polarization density. The considered medium consists of an assembly of identical atoms with Hamiltonian $H_{0}$, and the density matrix will be denoted by $\rho$. The Hamiltonian $H_{0}$ is given in diagonal form, $H_{0}=\hbar \operatorname{diag}\left(\omega_{1}, \omega_{2}, \ldots, \omega_{N}\right)$; thus we consider the general case of $N$-level identical atoms. The light matter coupling is described by

$$
\mathbf{P}=\mathcal{N} \operatorname{Tr}(\rho \boldsymbol{\mu})
$$

where $\boldsymbol{\mu}$ is the dipolar momentum operator and $\mathcal{N}$ is the atomic density. The evolution of $\rho$ is determined by

$$
i \hbar \frac{\partial \rho}{\partial t}=[H, \rho]
$$

so that the total Hamiltonian is

$$
H=H_{0}-\boldsymbol{\mu} \cdot \mathbf{E} .
$$

\section{B. Short-wave approximation regime}

We consider few-cycle optical solitons in the so-called short-wave approximation regime, i.e., the situation in which the transition (resonance) frequencies of the atoms are well below the optical frequencies, thus all transitions are in the infrared spectral domain. We next introduce the scaled variables $\tau$ (a retarded time) and $\zeta$,

$$
\tau=t-\frac{z}{V}, \quad \zeta=\varepsilon z,
$$

where $\varepsilon$ is a small parameter. Note that $\tau$ is not a slow variable, whereas the propagation variable $\zeta$ is. Thus the variable $\zeta$ gives account for long-distance propagation. We further assume a linearly polarized electrical field, perpendicular to the propagation direction $z$, say along $\mathbf{e}_{x}$, as $\mathbf{E}=E \mathbf{e}_{x}$, and expand $E$ in a power series of the small parameter $\varepsilon$ as $E=E^{(0)}+\varepsilon E^{(1)}+\cdots$. The polarization $\mathbf{P}=P \mathbf{e}_{x}$ and the density matrix $\rho$ are expanded in the same way.

The physical assumption is that all transition frequencies $\omega_{n}$ involved in the process are very low with respect to the typical wave frequency, i.e., we work in the so-called short-wave approximation regime. Hence all differences $\omega_{m n}=\omega_{m}-\omega_{n}$ are assumed to be small quantities of order $\varepsilon$. Formally, it is more convenient, and it does not modify the analysis, to consider that the frequencies $\omega_{n}$ themselves are small. Hence $H_{0}$ in Eq. (4) is replaced with $\varepsilon H_{0}$.

\section{Generic model}

Thus the corresponding series expansions of $E, P$, and $\rho$ are reported into the basic equations and the coefficients of identical powers of $\varepsilon$ on both sides of the equations are then identified.

\section{1. $\operatorname{Order} \varepsilon^{0}$}

Leading order $\varepsilon^{0}$ in Eq. (3) is

$$
i \hbar \frac{\partial}{\partial \tau} \rho^{(0)}=-\left[\mu E^{(0)}, \rho^{(0)}\right] .
$$

Then the polarization density at leading order is

$$
P^{(0)}=\mathcal{N} \operatorname{Tr}\left(\rho^{(0)} \mu\right) .
$$

From Eqs. (6) and (7), expanding the matrix operations, we get

$i \hbar \frac{\partial}{\partial \tau} P^{(0)}=-E^{(0)} \mathcal{N}\left(\sum_{n, m, v} \mu_{m v} \rho_{v n}^{(0)} \mu_{n m}-\sum_{n, m, v} \rho_{m v}^{(0)} \mu_{\nu n} \mu_{n m}\right)$.

Adequate permutation of the dummy subscripts in Eq. (8) shows that the two sums are identical and $P^{(0)}=0$.

The wave equation (1) at leading order $\varepsilon^{0}$ is

$$
\frac{1}{V^{2}} \frac{\partial^{2}}{\partial \tau^{2}} E^{(0)}=\frac{1}{c^{2}} \frac{\partial^{2}}{\partial \tau^{2}}\left(E^{(0)}+\frac{1}{\varepsilon_{0}} P^{(0)}\right) .
$$

Since $P^{(0)}=0$, Eq. (9) has nonzero solutions if $V=c$.

\section{2. $\operatorname{Order} \varepsilon^{1}$}

At order $\varepsilon^{1}$, Eq. (3) is

$$
i \hbar \frac{\partial}{\partial \tau} \rho^{(1)}=\left[H^{(0)}, \rho^{(0)}\right]-E^{(0)}\left[\mu, \rho^{(1)}\right]-E^{(1)}\left[\mu, \rho^{(0)}\right],
$$

or, in terms of matrix elements

$$
\begin{aligned}
i \hbar \frac{\partial}{\partial \tau} \rho_{m n}^{(1)}= & \hbar \omega_{m n} \rho_{m n}^{(0)}-E^{(0)} \sum_{\nu}\left(\mu_{m v} \rho_{v n}^{(1)}-\rho_{m \nu}^{(1)} \mu_{v n}\right) \\
& -E^{(1)} \sum_{\nu}\left(\mu_{m v} \rho_{v n}^{(0)}-\rho_{m v}^{(0)} \mu_{v n}\right)
\end{aligned}
$$

Here the fact that $H_{0}$ is diagonal has been used. By computing

$$
P^{(1)}=\mathcal{N} \operatorname{Tr}\left(\rho^{(1)} \mu\right)
$$

we find out that the last two terms in Eq. (11) give no contribution to $P^{(1)}$ as was previously seen when we have evaluated the right-hand side of Eq. (8). Thus we get

$$
\frac{\partial}{\partial \tau} P^{(1)}=-i \mathcal{N} \sum_{m n} \omega_{m n} \rho_{m n}^{(0)} \mu_{n m}
$$

The wave equation (1) at order $\varepsilon^{1}$ is

$$
\frac{1}{V^{2}} \frac{\partial^{2}}{\partial \tau^{2}} E^{(1)}-\frac{2}{V} \frac{\partial^{2}}{\partial \zeta \partial \tau} E^{(0)}=\frac{1}{c^{2}} \frac{\partial^{2}}{\partial \tau^{2}}\left(E^{(1)}+\frac{1}{\varepsilon_{0}} P^{(1)}\right) .
$$

Using $V=c$ and integrating once, with the assumption that the field vanishes at infinity, it reduces to

$$
\frac{\partial}{\partial \zeta} E^{(0)}=\frac{-1}{2 \varepsilon_{0} c} \frac{\partial}{\partial \tau} P^{(1)}
$$


Then we report Eq. (13) for $P^{(1)}$ into Eq. (15), which yields

$$
\frac{\partial}{\partial \zeta} E^{(0)}=\frac{i \mathcal{N}}{2 \varepsilon_{0} c} \sum_{m n} \omega_{m n} \rho_{m n}^{(0)} \mu_{n m} .
$$

The other equation is nothing else but Eq. (6), written in terms of matrix elements, as

$$
i \hbar \frac{\partial}{\partial \tau} \rho_{m n}^{(0)}=-E^{(0)} \sum_{\nu}\left(\mu_{m \nu} \rho_{v n}^{(0)}-\rho_{m \nu}^{(0)} \mu_{v n}\right) .
$$

Equations (16) and (17) yield the sought system in the general case.

Considering carefully Eqs. (16) and (17), it is seen that they do not reduce to a single $\mathrm{sG}$ equation as soon as two transitions are taken into account. Hence, in contrast to the long-wave approximation which is relevant for the ultraviolet resonances [51], the contribution of infrared resonances cannot be reduced in the general case to a form equivalent to the one which holds for a two-level system, which is sG. This is the first result of our investigation, in some sense a negative one. We may obviously consider the numerical solution of the full system (16) and (17) for any fixed number $N$ of levels, however it is a quite heavy task, and we are rather looking for simplified models. The question which arises now is whether a further approximation may reduce this full system to some simple generalization of $\mathrm{sG}$.

We will consider two special situations: the first one is the case of two independent transitions, which straightforwardly generalizes to an arbitrary number of independent transitions. A quite simple model, in which each transition gives rise to a sine term in the equation, is derived. Numerical analysis of the solutions in the case $N=2$ show that their behavior is not so far from that of the pure sG. Hence, if any interaction between the transitions is neglected, the problem of generalization of $\mathrm{sG}$ is solved. The question which remains is what happens when interaction between the transitions is taken into account? From the mathematical point of view, the problem is intractable and anything may happen. We thus will restrict to the interaction between two transitions only, i.e., a three-level system, in which we assumed that no transition between the two excited levels occurs. If both transitions involve the same transition dipolar momentum, the problem simplifies to a double-sG equation, which is in some sense one sine term form each of the nonlinear modes formed by the coupling between the two transitions.

\section{APPROXIMATION OF INDEPENDENT TRANSITIONS}

Let us consider a much simpler physical situation where a four-level system may interact with the light wave only through two independent transitions, i.e., we assume that the dipolar momentum operator has the form

$$
\mu=\left(\begin{array}{cccc}
0 & \mu_{12} & 0 & 0 \\
\mu_{12}^{*} & 0 & 0 & 0 \\
0 & 0 & 0 & \mu_{34} \\
0 & 0 & \mu_{34}^{*} & 0
\end{array}\right)
$$

Equation (16) reduces to

$$
\frac{\partial E^{(0)}}{\partial \zeta}=\frac{-\mathcal{N}}{\varepsilon_{0} c}\left(\omega_{12} Q_{12}+\omega_{34} Q_{34}\right),
$$

where we have set

$$
Q_{12}=\operatorname{Im}\left(\mu_{21} \rho^{12}\right), \quad Q_{34}=\operatorname{Im}\left(\mu_{43} \rho^{34}\right) .
$$

Defining the population differences as $w_{21}=\rho_{22}-\rho_{11}$ and $w_{43}=\rho_{44}-\rho_{33}$, the diagonal components of Eq. (17) reduce to

$$
\begin{aligned}
& \hbar \frac{\partial w_{21}}{\partial \tau}=-4 E^{(0)} Q_{12}, \\
& \hbar \frac{\partial w_{43}}{\partial \tau}=-4 E^{(0)} Q_{34} .
\end{aligned}
$$

The evolution of $Q_{12}$ and $Q_{34}$ is obtained from the off-diagonal component of Eq. (17) as

$$
\begin{aligned}
& \hbar \frac{\partial Q_{12}}{\partial \tau}=E^{(0)}\left|\mu_{12}\right|^{2} w_{21}, \\
& \hbar \frac{\partial Q_{34}}{\partial \tau}=E^{(0)}\left|\mu_{34}\right|^{2} w_{43} .
\end{aligned}
$$

The coupled equations (19) and (21)-(24) constitute the reduced system in the special case considered in this section. Recall that, in the case of a single transition for two-level systems, the analogous set of equations reduces to the sineGordon equation. Next let us try a change of variables analogous to the one which did allow this reduction, as

$$
\begin{aligned}
& w_{21}=A \cos u, \\
& w_{43}=B \cos v, \\
& Q_{12}=a A \sin u, \\
& Q_{34}=b B \sin v .
\end{aligned}
$$

Reporting into Eqs. (21) and (23), an adequate combination of both equations yields

$$
\hbar a \frac{\partial A}{\partial \tau}=E^{(0)} A \sin u \cos u\left(\left|\mu_{12}\right|^{2}-4 a^{2}\right)
$$

and consequently, if we choose $a=\left|\mu_{12}\right| / 2$, the function $A$ becomes constant. Obviously, so does $B$ if $b=\left|\mu_{34}\right| / 2$. Adequate combination of Eqs. (21)-(24) yield

$$
\begin{aligned}
& \frac{\partial u}{\partial \tau}=\frac{2}{\hbar}\left|\mu_{12}\right| A E^{(0)}, \\
& \frac{\partial v}{\partial \tau}=\frac{2}{\hbar}\left|\mu_{34}\right| B E^{(0)},
\end{aligned}
$$

i.e., both $u$ and $v$ are proportional to the $\tau$-antiderivative $\int^{\tau} E^{(0)} d \tau$ of the electric field. Then $Q_{12}$ and $Q_{34}$ are straightforwardly computed, and the evolution equation for 
the electric field becomes

$$
\begin{aligned}
\frac{\partial E^{(0)}}{\partial \zeta}= & \frac{-\mathcal{N}}{\varepsilon_{0} c}\left[\omega_{12}\left|\mu_{12}\right| \frac{A}{2} \sin \left(\frac{2}{\hbar}\left|\mu_{12}\right| A \int^{\tau} E^{(0)} d \tau\right)\right. \\
& \left.+\omega_{34}\left|\mu_{34}\right| \frac{B}{2} \sin \left(\frac{2}{\hbar}\left|\mu_{34}\right| B \int^{\tau} E^{(0)} d \tau\right)\right],
\end{aligned}
$$

The boundary conditions are as follows: we assume that, far from the pulse, the electric field is zero and the atoms are at thermal equilibrium. Let us denote by $\rho^{\text {th }}$ the density matrix at equilibrium; therefore $\rho^{\text {th }}$ is diagonal. Then $Q_{12}$ and $Q_{34}$ are equal to zero at infinity, and consequently so are $u$ and $v$. Then

$$
A=\lim _{\tau \longrightarrow-\infty} w_{21}=\rho_{22}^{\text {th }}-\rho_{11}^{\text {th }} .
$$

In the same way $B=\rho_{44}^{\text {th }}-\rho_{33}^{\text {th }}$, and the antiderivative $\int^{\tau} E^{(0)} d \tau$ of the electric field vanishes at infinity.

Let us introduce some reference electric field $E_{r}$ and define a dimensionless field $\psi=E_{0} / E_{r}$. Then, setting

$$
Z=\frac{\mathcal{N} \omega_{12}\left|\mu_{12}\right|\left(\rho_{22}^{\text {th }}-\rho_{11}^{\text {th }}\right)}{2 \varepsilon_{0} c E_{r}} \zeta
$$

and

$$
T=\frac{2}{\hbar}\left|\mu_{12}\right|\left(\rho_{22}^{\mathrm{th}}-\rho_{11}^{\mathrm{th}}\right) \tau,
$$

Eq. (32) reduces to the dimensionless form

$$
\frac{\partial \psi}{\partial Z}=\sin \left(\int_{-\infty}^{T} \psi d T^{\prime}\right)-q \sin \left(\lambda \int_{-\infty}^{T} \psi d T^{\prime}\right)
$$

where we have set

$$
\lambda=\frac{\left|\mu_{34}\right|\left(\rho_{44}^{\mathrm{th}}-\rho_{33}^{\mathrm{th}}\right)}{\left|\mu_{12}\right|\left(\rho_{22}^{\mathrm{th}}-\rho_{11}^{\mathrm{th}}\right)},
$$

and $q=\lambda \omega_{34} / \omega_{12}$.

Equation (36) is a generalization of the double-sineGordon equation, which is exactly Eq. (36) with $\lambda=2$. The above analysis straightforwardly generalizes to an arbitrary number $M$ of independent transitions, which formally would correspond to a dipolar momentum matrix presenting a set of $2 \times 2$ matrices

$$
\left(\begin{array}{cc}
0 & \mu_{j} \\
\mu_{j}^{*} & 0
\end{array}\right)
$$

along the diagonal, and zero everywhere else, and more physically to the assumption that the interaction between the transitions do not contribute. The evolution equation (32) then generalizes to

$$
\frac{\partial E^{(0)}}{\partial \zeta}=\frac{-\mathcal{N}}{\varepsilon_{0} c} \sum_{j=1}^{M} \omega_{j}\left|\mu_{j}\right| \frac{w_{j}}{2} \sin \left(\frac{2}{\hbar}\left|\mu_{j}\right| w_{j} \int^{\tau} E^{(0)} d \tau\right),
$$

in which $\omega_{j}$ and $w_{j}$ are the angular frequency and the population difference at thermal equilibrium for the $j$ th transition, respectively.

\section{INTERACTION BETWEEN TWO TRANSITIONS}

Consider now a three-level system, in which the two excited levels are not coupled together, i.e., the dipolar momentum matrix is

$$
\mu=\left(\begin{array}{ccc}
0 & \mu_{12} & \mu_{13} \\
\mu_{12}^{*} & 0 & 0 \\
\mu_{13}^{*} & 0 & 0
\end{array}\right) .
$$

Equation (16) reduces to

$$
\frac{\partial E^{(0)}}{\partial \zeta}=\frac{-\mathcal{N}}{\varepsilon_{0} c}\left(\omega_{12} Q_{12}+\omega_{13} Q_{13}\right)
$$

with the $Q_{m n}$ defined in an analogous way as in Eq. (20). The diagonal components of Eq. (17) reduce to

$$
\begin{aligned}
& \hbar \frac{\partial \rho_{22}}{\partial \tau}=-2 E^{(0)} Q_{12}, \\
& \hbar \frac{\partial \rho_{33}}{\partial \tau}=-2 E^{(0)} Q_{13},
\end{aligned}
$$

and

$$
\rho_{11}=1-\rho_{22}-\rho_{33} .
$$

The off-diagonal components are written as

$i \hbar \frac{\partial}{\partial \tau} \mu_{12}^{*} \rho_{12}=-E^{(0)}\left[\left|\mu_{12}\right|^{2}\left(\rho_{22}-\rho_{11}\right)+\mu_{12}^{*} \mu_{13} \rho_{32}\right]$,

an analogous equation can be written for $\rho_{13}$, and

$$
i \hbar \frac{\partial}{\partial \tau} \mu_{13}^{*} \mu_{12} \rho_{23}=-E^{(0)}\left(\mu_{13}^{*}\left|\mu_{12}\right|^{2} \rho_{13}-\mu_{12}\left|\mu_{13}\right|^{2} \rho_{12}^{*}\right) \text {. }
$$

We set $\mu_{1 n}^{*} \rho_{1 n}=P_{1 n}+i Q_{1 n}$, for $n=2,3$ and $\mu_{13}^{*} \mu_{12} \rho_{23}=$ $R+i S$, with $P_{1 n}, Q_{1 n}, R$, and $S$ real. After reporting these definitions into Eqs. (44) and (45), and separating real and imaginary parts, it is seen that $S, P_{12}$, and $P_{13}$ are zero, and we get the equations

$$
\begin{aligned}
& \hbar \frac{\partial R}{\partial \tau}=-E^{(0)}\left(\left|\mu_{12}\right|^{2} Q_{13}+\left|\mu_{13}\right|^{2} Q_{12}\right), \\
& \hbar \frac{\partial Q_{12}}{\partial \tau}=E^{(0)}\left(\left|\mu_{12}\right|^{2}\left(\rho_{22}-\rho_{11}\right)+R\right), \\
& \hbar \frac{\partial Q_{13}}{\partial \tau}=E^{(0)}\left(\left|\mu_{13}\right|^{2}\left(\rho_{33}-\rho_{11}\right)+R\right),
\end{aligned}
$$

which, together with Eqs. (40)-(43), yield the sought after system.

It is seen that this set of equations remains a complicated one. However, if, in addition, the two transitions have the same transition dipolar momentum, i.e., if

$$
\left|\mu_{12}\right|=\left|\mu_{13}\right|=\mu,
$$

then it can be considerably simplified.

Let us set $Q_{ \pm}=Q_{12} \pm Q_{13}$, and $\rho_{+}=\rho_{33}+\rho_{22}+\alpha$ with $\alpha$ some constant, and $\rho_{-}=\rho_{33}-\rho_{22}$.

Then Eqs (40)-(43) and (46)-(48) become

$$
\begin{gathered}
\hbar \frac{\partial R}{\partial \tau}=-E^{(0)} \mu^{2} Q_{+}, \\
\hbar \frac{\partial Q_{+}}{\partial \tau}=E^{(0)}\left[\mu^{2}\left(3 \rho_{+}-3 a-2\right)+2 R\right],
\end{gathered}
$$




$$
\begin{aligned}
& \hbar \frac{\partial Q_{-}}{\partial \tau}=E^{(0)} \mu^{2} \rho_{-}, \\
& \hbar \frac{\partial \rho_{ \pm}}{\partial \tau}=-2 E^{(0)} Q_{ \pm},
\end{aligned}
$$

and

$$
\frac{\partial E^{(0)}}{\partial \zeta}=\frac{-\mathcal{N}}{\varepsilon_{0} c}\left(\omega_{+} Q_{+}+\omega_{-} Q_{-}\right),
$$

with

$$
\omega_{ \pm}=\frac{\omega_{13} \pm \omega_{12}}{2} .
$$

Comparing (50) and (54), it is seen that

$$
R=\frac{\mu^{2}}{2} \rho_{+}+K,
$$

where $K$ is some constant. Equation (51) reduces to

$$
\hbar \frac{\partial Q_{+}}{\partial \tau}=3 E^{(0)} \mu^{2} \rho_{+}
$$

if we set the arbitrary constant $\alpha$ to

$$
\alpha=-\frac{2}{3}+\frac{2 K}{3 \mu^{2}} \text {. }
$$

Consider then the change of variables analogous to (25)-(28):

$$
\begin{gathered}
\rho_{ \pm}=A_{ \pm} \cos u_{ \pm}, \\
Q_{ \pm}=b_{ \pm} A_{ \pm} \sin u_{ \pm} .
\end{gathered}
$$

Reporting (59) and (60) into (57), (52), and (53), it is seen that, if

$$
b_{+}=\sqrt{2} \mu, \quad \text { and } \quad b_{-}=\frac{\mu}{\sqrt{2}},
$$

the equations for $A_{ \pm}$simplify to $\partial A_{ \pm} / \partial_{\tau}=0$. Then the other equations are solved straightforwardly to yield

$$
\begin{aligned}
& u_{+}=\frac{2 \sqrt{2} \mu}{\hbar} \int_{-\infty}^{\tau} E^{(0)} d \tau, \\
& u_{-}=\frac{\sqrt{2} \mu}{\hbar} \int_{-\infty}^{\tau} E^{(0)} d \tau .
\end{aligned}
$$

Note that $u_{ \pm}$vanish at infinity since the wave does. The whole set of equations reduces to the evolution equation for the electric field, which becomes

$$
\begin{aligned}
\frac{\partial E^{(0)}}{\partial \zeta}= & \frac{-\mathcal{N}}{\varepsilon_{0} c}\left[\omega_{+} \mu \sqrt{2} A_{+} \sin \left(\frac{2 \sqrt{2} \mu}{\hbar} \int_{-\infty}^{\tau} E^{(0)} d \tau\right)\right. \\
& \left.+\omega_{-} \frac{\mu}{\sqrt{2}} A_{-} \sin \left(\frac{\sqrt{2} \mu}{\hbar} \int_{-\infty}^{\tau} E^{(0)} d \tau\right)\right] .
\end{aligned}
$$

The conditions at infinity show directly that $A_{+}=\rho_{22}^{\text {th }}+$ $\rho_{33}^{\text {th }}+\alpha$, while $R$ on one hand tends to 0 , and on the other hand, tends to $K+\mu^{2} A_{+} / 2$, which gives the value of $K$ and then

$$
A_{+}=\frac{3}{4}\left(\rho_{22}^{\text {th }}+\rho_{33}^{\text {th }}\right)-\frac{1}{2},
$$

while

$$
A_{-}=\rho_{33}^{\text {th }}-\rho_{22}^{\text {th }} \text {. }
$$

By setting the dimensionless quantities

$$
\begin{gathered}
\psi=\frac{E^{(0)}}{E_{r}}, \\
T=\frac{\sqrt{2} \mu E_{r}}{\hbar} \tau, \\
Z=\frac{\mathcal{N} \mu \omega_{-}}{\varepsilon_{0} c E_{r}} \frac{\sqrt{2}}{2}\left(\rho_{22}^{\mathrm{th}}-\rho_{33}^{\mathrm{th}}\right) \zeta,
\end{gathered}
$$

we reduce Eq. (64) to the dimensionless form

$$
\frac{\partial \psi}{\partial Z}=\sin \left(\int_{-\infty}^{T} \psi d T^{\prime}\right)+q \sin \left(2 \int_{-\infty}^{T} \psi d T^{\prime}\right),
$$

where we have set

$$
q=\frac{\left(\omega_{13}+\omega_{12}\right)\left(\rho_{22}^{\text {th }}+\rho_{33}^{\text {th }}-2 \rho_{11}^{\text {th }}\right)}{2\left(\omega_{13}-\omega_{12}\right)\left(\rho_{33}^{\text {th }}-2 \rho_{22}^{\text {th }}\right)} .
$$

The obtained dimensionless equation (70) is the so-called double-sG equation, which was studied in detail in the mathematical literature; see, e.g., Ref. [53].

Equation (71), or in physical units, Eq. (64), involves two sine terms, one of which involves the sum frequency $\omega_{13}+\omega_{12}$, the other the difference frequency $\omega_{13}-\omega_{12}$. They seem to arise from some kind of symmetric and antisymmetric hybridization of the linear modes due to their nonlinear coupling. Unfortunately, our mathematical analysis is restricted to a specific situation, and cannot be generalized in an exact way. However, we may expect that such a generalization, introduced in a phenomenological way, might be relevant in many real situations.

\section{BREATHER SOLUTION TO THE DOUBLE-SG EQUATION}

The double-sG equation (36) with $\lambda=2$ (or equivalently $1 / 2$ ) has been often studied in the literature; however it does not belong to the known set of completely integrable nonlinear partial differential equations but it possesses a set of analytical solutions. In Ref. [53] both a multiple sG equation and a double-sG equation in the framework of SIT were derived. Their soliton solutions were studied in Ref. [53], especially two-lumped ones. Later, in Ref. [54], although its title involves SIT, a physical situation closer to that of the present paper was considered. The solitons discussed in Ref. [54] are two-humped ones, as in Ref. [53]. In Ref. [55] a perturbation theory was developed for the double-sG equation, in order to treat additional small terms added to it, whereas in Ref. [56] a formal expansion method was proposed to find analytical solutions to sG-type equations, with specific application to the double-sG equation. Also, in Ref. [57] a set of exact solutions to the double-sG equation was given.

However, while both kinds of solitary wave type solutions and periodic type solutions have been considered in the above-mentioned works, solutions in form of ultrashort wave packets, i.e., breather-type ones, have not. The latter are the relevant solutions for few-cycle optical soliton propagation in such physical settings. Numerical resolution of Eq. (36) [or Eq. (70)] was performed using a standard fourth-order Runge-Kutta scheme in the Fourier domain. The antiderivative is obtained by division with $2 i \pi \nu$ where the frequency $v$ is the 


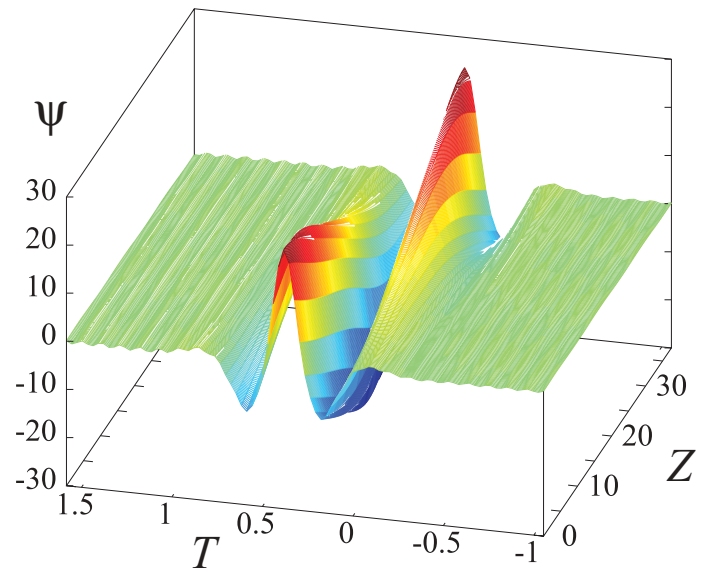

FIG. 1. (Color online) One period of a breather of the standard double-sG equation, for $\lambda=2, q=0.2$.

Fourier variable, under the assumption that the mean value, obtained at $v=0$, is zero. Then the nonlinear, sine terms are obtained by means of one inverse and one direct fast-Fourier transforms at each substep of the Runge-Kutta scheme.

Breathers form spontaneously from a sinusoidal input with a Gaussian envelope. However, during pulse reshaping, an appreciable amount of radiation is emitted. The question of whether the breather formed this way is stable then arises. In order to evaluate its stability, an input datum close enough to a breather is built. Note that, in contrast to the solitary wave, the breather is not the solution of an ordinary differential equation. It is not stationary but evolves periodically, together with moving at some nonlinear group velocity different of the linear one (see Fig. 1). To isolate a breather, we used the following procedure: starting from a Gaussian pulse, the evolution is computed over some distance (we used 226.5), then the radiation is removed by replacing with zero the field at some distance from the maximum (we used a window width of 7.5), then the field is multiplied with some constant, so that the energy is the same as the initial one. This operation is iterated until the energy loss decreases below some fixed precision (we

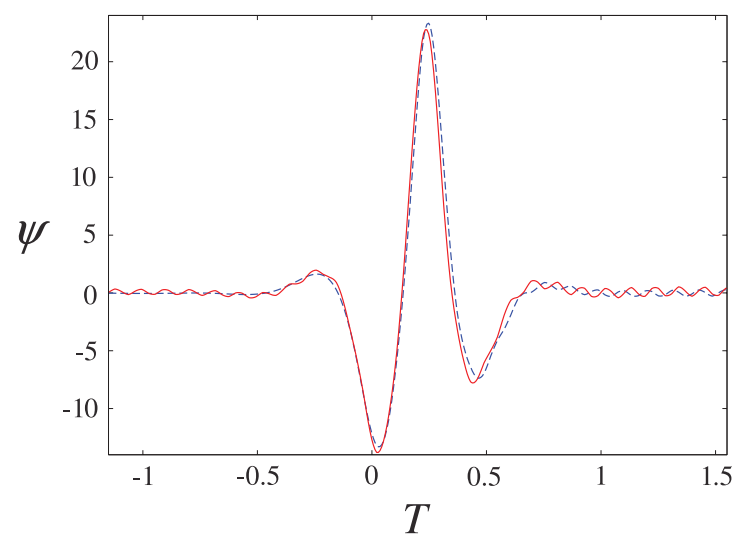

FIG. 2. (Color online) Propagation of a breather of the standard double-sG equation, for $\lambda=2, q=0.2$. Dashed line: a numerically computed breather as initial data; solid line: after propagation over $Z>10000$ (for the same value of the breather phase and compensation of group velocity).

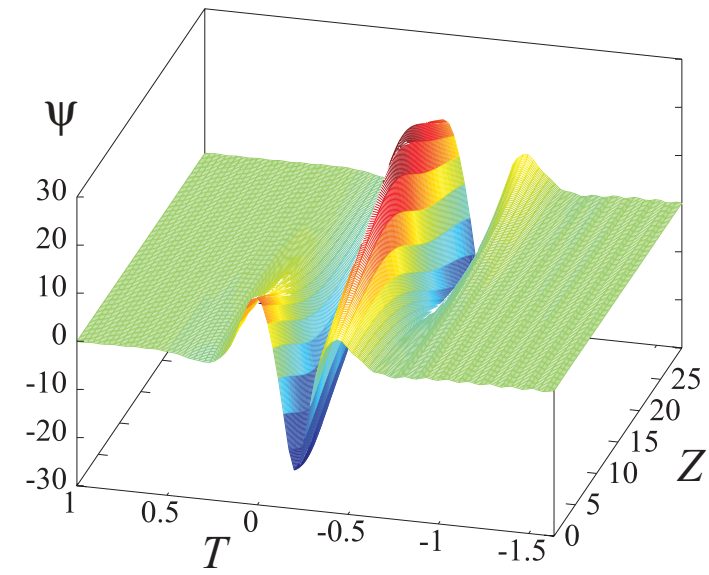

FIG. 3. (Color online) One period of a breather of the generalized double-sG equation, for $\lambda=\sqrt{3}, q=0.4$.

used $0.1 \%$ ). Although very slowly, it converges. The result can be considered as a good numerical approximation of the breather. It is used as input and its evolution is computed over a very long propagation distance $(Z=10000)$. Then, the evolution of the output is monitored over a short distance, with a high resolution in $Z$, which yields the results shown in Fig. 1.

Finally, we select within this monitoring the exact propagation distance at which the breather phase (carrier-envelope phase) is the same as at the input, and move it along the $T$ axis so that the pulse location coincides with that of the input. This yields the results displayed in Fig. 2. Thus the robustness of the breather-type solution is demonstrated in this way. The question also arises whether stable breathers also exist for values $\lambda$ of the frequency ratio of the two sine functions in the double-sG equation, other than 2, i.e., for a generalized double-sG equation, which describes a much broader physical situation. We consider a value with the same order of magnitude, but such that both frequencies are not commensurable, e.g., we take $\lambda=\sqrt{3}$. The corresponding computations yield analogous results, shown on Figs. 3 and 4 .

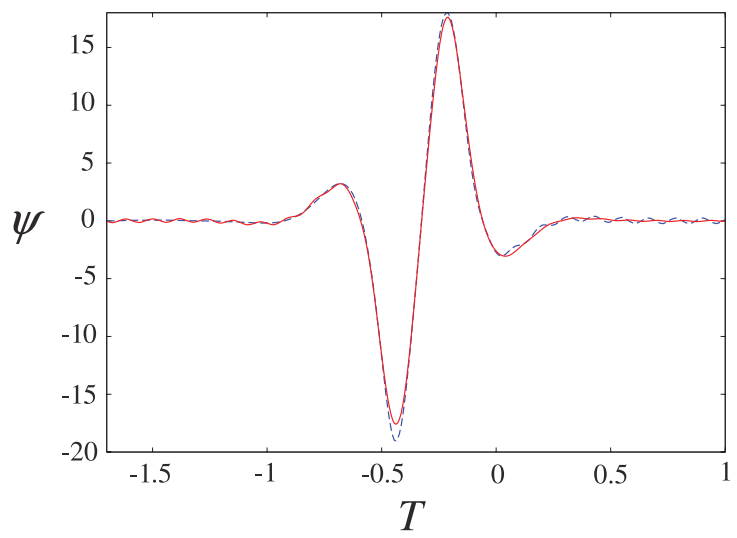

FIG. 4. (Color online) Propagation of a breather of the generalized double-sG equation, for $\lambda=\sqrt{3}, q=0.4$. Dashed line: a numerically computed breather as initial data; solid line: after propagation over $Z>10000$ (for the same value of the breather phase and compensation of group velocity). 
Thus the existence of a stable breather solution is also evidenced in the most general case.

\section{CONCLUSIONS}

In conclusion, we have derived a generic model beyond the slowly varying envelope approximation of the nonlinear Schrödinger-type evolution equations, describing the propagation of few-optical cycle pulses in a generic medium. We used the density-matrix formalism for a general Hamiltonian of multilevel identical atoms, and assumed that all transition frequencies of the medium are well below the typical wave frequency, i.e., only the contribution of infrared transitions is taken into account. Then the application of the reductive perturbation formalism in the short-wave approximation, up to the second order in some small perturbation parameter, allowed us to give a rigorous derivation of a coupled system of nonlinear partial differential equations describing FCP evolution in such systems. However, this model remains very complicated in the general case. If any coupling or interaction between transitions is neglected, it simplifies to a sG-type equation, involving one sine term for each transition. We specifically analyzed the case of two independent transitions, where the general system can be reduced to a generalized double-sine-Gordon equation.

The coupling between transitions is investigated only in the most simple case, of two transitions in three-level atoms, and assuming that both oscillators have exactly the same weight, i.e., the same dipolar momentum. In this special case, the model reduces to a standard double-sine-Gordon equation. Direct numerical simulations showed the formation of robust breather-type solutions of both the standard and the generalized double-sine-Gordon equations from sinusoidal inputs with Gaussian envelopes.

The present work might be extended in two directions. First, in a real optical medium, a broad transparency range is required, and hence all atomic transitions must be far enough from the pulse central frequency. However, in such materials not all transitions belong to the infrared spectral domain. Note that the contribution of the ultraviolet transitions in the general situation of multilevel atoms was considered in a recent work [50] and a modified Korteweg-de Vries model was put forward by means of a long-wave approximation applied to the same general quantum model. However, a fully realistic model for ultrashort pulse propagation might be obtained by putting together the results yielded by both long- and short-wave approaches. Second, the present study, which was restricted to $(1+1)$ dimensions, can be extended to $(2+1)$ dimensions by incorporating into the generic model a transverse spatial coordinate; thus the formation of ultrashort spatiotemporal optical solitons (alias "light bullets") [58] can be investigated in the more general setting of a collection of multilevel identical atoms.

\section{ACKNOWLEDGMENTS}

The work of D.M. was supported in part by a Senior Chair Grant from the Région Pays de Loire, France. Support from the Romanian Ministry of Education and Research (Project PN-II-ID-PCE-2011-3-0083) is also acknowledged.
[1] L. Gallmann, D. H. Sutter, N. Matuschek, G. Steinmeyer, U. Keller, C. Iaconis, and I. A. Walmsley, Opt. Lett. 24, 1314 (1999); U. Morgner, F. X. Kärtner, S. H. Cho, Y. Chen, H. A. Haus, J. G. Fujimoto, E. P. Ippen, V. Scheuer, G. Angelow, and T. Tschudi, ibid. 24, 411 (1999); D. H. Sutter, G. Steinmeyer, L. Gallmann, N. Matuschek, F. Morier-Genoud, U. Keller, V. Scheuer, G. Angelow, and T. Tschudi, ibid. 24, 631 (1999); A. Shirakawa, I. Sakane, M. Takasaka, and T. Kobayashi, App. Phys. Lett. 74, 2268 (1999).

[2] T. Brabek and F. Krausz, Rev. Mod. Phys. 72, 545 (2000).

[3] E. Goulielmakis, M. Schultze, M. Hofstetter, V. S. Yakovlev, J. Gagnon, M. Uiberacker, A. L. Aquila, E. M. Gullikson, D. T. Attwood, R. Kienberger, F. Krausz, and U. Kleineberg, Science 320, 1614 (2008).

[4] M. Drescher, M. Hentschel, R. Kienberger, G. Tempea, C. Spielmann, G. A. Reider, P. B. Corkum, and F. Krausz, Science 291, 1923 (2001); M. Hentschel, R. Kienberger, C. Spielmann, G. A. Reider, N. Milosevic, T. Brabec, P. Corkum, U. Heinzmann, M. Drescher, and F. Krausz, Nature (London) 414, 509 (2001); P. M. Paul, E. S. Toma, P. Breger, G. Mullot, F. Augé, P. Balcou, H. G. Muller, and P. Agostini, Science 292, 1689 (2001).

[5] A. Scrinzi, M. Yu. Ivanov, R. Kienberger, and D. M. Villeneuve, J. Phys. B 39, R1 (2006).
[6] F. Krausz and M. Ivanov, Rev. Mod. Phys. 81, 163 (2009).

[7] A. Baltuska, Th. Udem, M. Uiberacker, M. Hentschel, E. Goulielmakis, Ch. Gohle, R. Holzwarth, V. S. Yakovlev, A. Scrinzi, T. W. Hänsch, and F. Krausz, Nature (London) 421, 611 (2003).

[8] H.-C. Wu and J. Meyer-ter-Vehn, Nat. Photonics 6, 304 (2012).

[9] G. Krauss, S. Lohss, T. Hanke, A. Sell, S. Eggert, R. Huber, and A. Leitenstorfer, Nat. Photonics 4, 33 (2010).

[10] M. Bock, J. Jahns, and R. Grunwald, Opt. Lett. 37, 3804 (2012); K. Yamane, Y. Toda, and R. Morita, Opt. Express 20, 18986 (2012).

[11] G. A. Mourou, N. J. Fisch, V. M. Malkin, Z. Toroker, E. A. Khazanov, A. M. Sergeev, T. Tajima, and B. Le Garrec, Opt. Commun. 285, 720 (2012).

[12] X. Tan, X. Fan, Y. Yang, and D. Tong, J. Mod. Opt. 55, 2439 (2008); N. N. Rosanov, V. E. Semenov, and N. V. Vyssotina, Laser Phys. 17, 1311 (2007); Quantum Electron. 38, 137 (2008); A. Nazarkin, Phys. Rev. Lett. 97, 163904 (2006); A. I. Maimistov, Quantum Electron. 40, 756 (2010); Ni Cui and M. A. Macovei, New J. Phys. 14, 093031 (2012).

[13] T. Brabec and F. Krausz, Phys. Rev. Lett. 78, 3282 (1997); M. V. Tognetti and H. M. Crespo, J. Opt. Soc. Am. B 24, 1410 (2007); A. A. Voronin and A. M. Zheltikov, Phys. Rev. A 78, 063834 (2008); A. Kumar and V. Mishra, ibid. 79, 063807 (2009); P. Kinsler and G. H. C. New, ibid. 67, 023813 (2003); A. A. 
Zozulya, S. A. Diddams, and T. S. Clement, ibid. 58, 3303 (1998); N. Akozbek, M. Scalora, C. M. Bowden, and S. L. Chin, Opt. Commun. 191, 353 (2001); J. E. Rothenberg, Opt. Lett. 17, 1340 (1992); J. K. Ranka and A. L. Gaeta, ibid. 23, 534 (1998).

[14] E. M. Belenov and A. V. Nazarkin, Pis'ma Zh. Eksp. Teor. Fiz. 51, 252 (1990) [JETP Lett. 51, 288 (1990)].

[15] A. I. Maimistov and S. O. Elytin, J. Mod. Opt. 39, 2201 (1992).

[16] A. E. Kaplan and P. L. Shkolnikov, Phys. Rev. Lett. 75, 2316 (1995).

[17] M. A. Porras, Phys. Rev. A 60, 5069 (1999).

[18] S. V. Sazonov, Zh. Eksp. Teor. Fiz. 119, 419 (2001) [JETP 92, 361 (2001)].

[19] A. I. Maimistov, Opt. Spektrosk. 76, 636 (1994) [Opt. Spectrosc. 76, 569 (1994)].

[20] I. V. Mel'nikov, D. Mihalache, F. Moldoveanu, and N.-C. Panoiu, Phys. Rev. A 56, 1569 (1997).

[21] I. V. Mel'nikov, D. Mihalache, and N.-C. Panoiu, Opt. Commun. 181, 345 (2000).

[22] H. Leblond and F. Sanchez, Phys. Rev. A 67, 013804 (2003).

[23] I. V. Mel'nikov, H. Leblond, F. Sanchez, and D. Mihalache, IEEE J. Sel. Top. Quantum Electron. 10, 870 (2004); H. Leblond, F. Sanchez, I. V. Mel'nikov, and D. Mihalache, Math. Comput. Simul. 69, 378 (2005).

[24] H. Leblond, S. V. Sazonov, I. V. Mel'nikov, D. Mihalache, and F. Sanchez, Phys. Rev. A 74, 063815 (2006).

[25] H. Leblond, I. V. Mel'nikov, and D. Mihalache, Phys. Rev. A 78, 043802 (2008).

[26] H. Leblond and D. Mihalache, Phys. Rev. A 79, 063835 (2009); Rom. Rep. Phys. 63, 1254 (2011); Phys. Reports, doi: 10.1016/j.physrep.2012.10.006.

[27] S. A. Skobelev, D. V. Kartashov, and A. V. Kim, Phys. Rev. Lett. 99, 203902 (2007).

[28] Sh. Amiranashvili, A. G. Vladimirov, and U. Bandelow, Phys. Rev. A 77, 063821 (2008).

[29] T. Schäfer and C. E. Wayne, Physica D 196, 90 (2004).

[30] N. L. Tsitsas, T. P. Horikis, Y. Shen, P. G. Kevrekidis, N. Whitaker, and D. J. Frantzeskakis, Phys. Lett. A 374, 1384 (2010).

[31] Y. Shen, N. Whitaker, P. G. Kevrekidis, N. L. Tsitsas, and D. J. Frantzeskakis, Phys. Rev. A 86, 023841 (2012).

[32] M. Kolesik, J. V. Moloney, and M. Mlejnek, Phys. Rev. Lett. 89, 283902 (2002); M. Kolesik and J. V. Moloney, Phys. Rev. E 70, 036604 (2004); M. Kolesik, P. Jakobsen, and J. V. Moloney, Phys. Rev. A 86, 035801 (2012).

[33] A. V. Husakou and J. Herrmann, Phys. Rev. Lett. 87, 203901 (2001).

[34] N. N. Akhmediev, I. V. Mel'nikov, and A. V. Nazarkin, Kratk. Soobshch. Fiz. FIAN 2, 49 (1989) [Sov. Phys. Lebedev. Inst. Rep. 2, 66 (1989)].
[35] E. D. Farnum and J. Nathan Kutz, Opt. Lett. 35, 3033 (2010)

[36] E. V. Kazantseva and A. I. Maimistov, Phys. Lett. A 263, 434 (1999); E. V. Kazantseva, A. I. Maimistov, and B. A. Malomed, Opt. Commun. 188, 195 (2001); H. Leblond, Phys. Rev. A 78, 013807 (2008).

[37] L. Bergé and S. Skupin, Phys. Rev. Lett. 100, 113902 (2008).

[38] H. Leblond, D. Kremer, and D. Mihalache, Phys. Rev. A 80, 053812 (2009).

[39] H. Leblond, D. Kremer, and D. Mihalache, Phys. Rev. A 81, 033824 (2010); H. Leblond and D. Mihalache, ibid. 81, 063815 (2010).

[40] N. N. Rosanov, V. V. Kozlov, and S. Wabnitz, Phys. Rev. A 81, 043815 (2010); H. Leblond and D. Mihalache, J. Phys. A: Math. Theor. 43, 375205 (2010).

[41] V. V. Kozlov, N. N. Rosanov, C. De Angelis, and S. Wabnitz, Phys. Rev. A 84, 023818 (2011).

[42] A. Pusch, J. M. Hamm, and O. Hess, Phys. Rev. A 84, 023827 (2011).

[43] X.-T. Xie and M. A. Macovei, Phys. Rev. Lett. 104, 073902 (2010).

[44] A. V. Kim and S. A. Skobelev, Phys. Rev. A 83, 063832 (2011)

[45] H. Leblond, H. Triki, F. Sanchez, and D. Mihalache, Phys. Rev. A 83, 063802 (2011); Opt. Commun. 285, 356 (2012); H. Leblond, H. Triki, and D. Mihalache, Phys. Rev. A 84, 023833 (2011).

[46] A. A. Drozdov, S. A. Kozlov, A. A. Sukhorukov, and Y. S. Kivshar, Phys. Rev. A 86, 053822 (2012).

[47] M. Kolesik, P. Jakobsen, and J. V. Moloney, Phys. Rev. A 86, 035801 (2012).

[48] P. Whalen, J. V. Moloney, A. C. Newell, K. Newell, and M. Kolesik, Phys. Rev. A 86, 033806 (2012).

[49] Zhenya Yan, J. Phys. A: Math. Theor. 45, 444035 (2012).

[50] H. Triki, H. Leblond, and D. Mihalache, Opt. Commun. 285, 3179 (2012).

[51] H. Leblond, H. Triki, and D. Mihalache, Phys. Rev. A 85, 053826 (2012).

[52] H. Leblond, J. Phys. B 41, 043001 (2008).

[53] R. K. Bullough and P. J. Caudrey, Rocky Mountain J. Math. 8, 53 (1978).

[54] A. Yu. Parkhomenko and S. V. Sazonov, Zh. Eksp. Teor. Fiz. 114, 1595 (1998) [JETP 87, 864 (1998)].

[55] C. A. Popov, Wave Motion 42, 309 (2005).

[56] Zhenya Yan, Chaos Solitons Fractals 23, 767 (2005).

[57] M. Wang and X. Li, Chaos Solitons Fractals 27, 477 (2006).

[58] B. A. Malomed, D. Mihalache, F. Wise, and L. Torner, J. Opt. B 7, R53 (2005); D. Mihalache, Proc. Romanian Acad. A 11, 142 (2010); Rom. Rep. Phys. 63, 9 (2011); Rom. J. Phys. 57, 352 (2012). 